La revue La revue pour l'histoire du CNRS

\title{
La Société algérienne de chimie
}

Processus d'émergence et de développement d'une société savante

(1962-1994)

Yamina Bettahar

\section{(2) OpenEdition}

Journals

Édition électronique

URL : https://journals.openedition.org/histoire-cnrs/1358

DOI : 10.4000/histoire-cnrs. 1358

ISSN : 1955-2408

Éditeur

CNRS Éditions

\section{Édition imprimée}

Date de publication : 3 mai 2005

ISBN : 978-2-271-06250-5

ISSN : 1298-9800

\section{Référence électronique}

Yamina Bettahar, «La Société algérienne de chimie », La revue pour l'histoire du CNRS [En ligne], 12 | 2005, mis en ligne le 03 mai 2007, consulté le 20 mai 2021. URL : http://journals.openedition.org/ histoire-cnrs/1358; DOI : https://doi.org/10.4000/histoire-cnrs.1358

Ce document a été généré automatiquement le 20 mai 2021.

Comité pour l'histoire du CNRS 


\title{
La Société algérienne de chimie
}

\author{
Processus d'émergence et de développement d'une société savante
}

(1962-1994)

\section{Yamina Bettahar}

Introduction

1 La chimie est une discipline-phare par ses applications industrielles. Elle est devenue une des composantes majeures des activités humaines. Selon le chimiste français JeanMarie Lehn - prix Nobel de chimie en 1987 -, elle permet « à l'homme de connaître la matière et ses transformations, d'agir sur elle, de la modifier et d'en inventer de nouvelles manifestations $»^{1}$. Aussi joue t-elle un rôle central par son importance économique et son omniprésence dans notre vie quotidienne. Aujourd'hui, ce qui caractérise la chimie, c'est qu'elle couvre un vaste champ qui s'étend « des confins de la physique à ceux de la biologie » et c'est dans cette discipline que la distinction entre science fondamentale dite "pure » et science appliquée ou «finalisée " prendrait toute sa signification et qu'apparaîtraient des interactions encore plus fortes que dans les autres disciplines scientifiques.

2 En Algérie, bien que née dans le giron de la faculté des sciences de l'université coloniale $^{2}$ et contrairement à la médecine, la géologie ou l'agriculture qui furent l'objet d'enjeux importants et d'une instrumentalisation précoce, la chimie a connu un rôle tout à fait accessoire, les «chimistes" coloniaux étant peu favorables au développement de cette discipline localement ${ }^{3}$. C'est seulement après l'indépendance de l'Algérie que la chimie a connu son véritable essor. Cela reflète le rôle désormais assigné à la science dans le développement économique et social du pays. Ce revirement est sans doute lié au projet modernisateur et à la stratégie de développement accéléré mise en chantier à la fin des années 1960. Celle-ci est fondée sur la récupération et la valorisation des richesses naturelles au rang desquelles les hydrocarbures et les industries dérivées occupent une place de choix. Le processus de modernisation devient une affaire d'État et aboutit à de multiples transformations dont profitent largement les champs académique et scientifique : massification de l'accès aux études scientifiques, démocratisation et algérianisation des personnels par une politique de formation accrue à l'étranger, refonte des programmes de formation 
spécialisée et de la politique scientifique, re-création d'un dispositif institutionnel de recherche plus adapté aux exigences du développement, coopération culturelle et scientifique internationale dans laquelle la France occupe une place privilégiée en raison de l'héritage linguistique et de la densité des relations historiques ${ }^{4}$ et se traduit par l'accueil en France d'étudiants et d'enseignants mais contribue également largement à la formation sur place des compétences scientifiques et techniques algériennes.

3 Prenant pour exemple la fondation d'une société savante et d'une revue scientifique en chimie, cet article se propose d'analyser et de comprendre ces mutations. Après avoir présenté à grand trait l'évolution et le développement du système national de recherche, dont le mode d'organisation de la recherche et du rôle de l'État restent fortement calqués sur le modèle centralisé de la France, nous nous attacherons ensuite à l'étude des conditions d'émergence et de développement d'une communauté scientifique au travers de la Société algérienne de chimie (Sac), de ses réseaux de sociabilité scientifique et enfin de ses partenariats internationaux, principalement avec la France.

Le système national de recherche

4 Durant la décennie 1970-1980, le discours dominant sur l'enseignement supérieur a un fondement économique et technique. Au début des années 1970, le système d'enseignement supérieur algérien entre alors dans un vaste processus de réformes fondamentales appelé à l'intégrer dans le mouvement d'ensemble de la société et de l'économie. Selon le texte portant réforme de l'enseignement supérieur (1971), l'Université doit être le reflet des mutations technologiques du secteur productif. Lieu de production et de reproduction des connaissances scientifiques et technologiques, l'institution universitaire doit contribuer à la formation du personnel qualifié dont a besoin le pays pour jeter les bases du décollage économique. «L'option scientifique et technique " fait l'objet d'une série de textes législatifs et réglementaires qui en organisent et définissent les contours dès $1971^{5}$. Le pouvoir politique soutient les actions de restructuration de l'Université et du système éducatif dans son ensemble.

La science au service du politique

Dans l'Algérie indépendante, les relations du pouvoir et de la science ont une histoire récente. La politique de la science est un paradigme qui date seulement des années 1970.

6 Si l'intervention de l'État en matière de recherche scientifique prend une forme dirigiste et organisée seulement à partir des années 1970, les enjeux autour de son contrôle ont été posés dès 1962. Dès ce moment, l'État prend conscience du rôle de la science et de la technologie dans la réponse aux besoins que pourraient faire naître les programmes de développement économique et social. Dans le projet modernisateur mis en œuvre par l'État dans les années 1970, la science et la technologie jouent un rôle important dans la politique d'industrialisation et la construction de groupes professionnels. Des moyens sont alors mobilisés par les politiques pour la création de pôles scientifiques et technologiques, le développement de la recherche universitaire fondamentale et/ou appliquée, la création d'organismes de recherche, la formation de scientifiques, l'octroi d'aides à la recherche et à l'innovation technologique. Pour ce faire, un appareil scientifique est mis en place. 
7 Sans entrer dans les détails, nous allons essayer de le présenter dans ses grandes lignes. D'emblée, on sait que cet appareil a eu un démarrage prometteur mais un certain nombre de facteurs ont limité son fonctionnement.

8 La politique de la science, nous dit Jean-Jacques Salomon, c'est «l'ensemble des dispositions prises par un gouvernement, d'une part pour encourager le développement de la recherche scientifique et technique et, d'autre part, pour tirer parti des résultats de la recherche à des fins de politique générale $»^{6}$; donc une politique pour et par la science. Qu'en est-il en Algérie?

La période de gestion de l'héritage colonial

Dans les années 1962-1971, la création du Conseil de la recherche scientifique est un compromis dans les rapports franco-algériens. Au plan général, si les rapports entre l'Algérie et la France ont toujours été complexes, passionnels et passionnés, il convient tout de même de souligner une volonté indéniable de maintenir les liens et d'entreprendre des actions communes. Cette volonté se traduisit par la définition d'un nouveau rapport bilatéral, voire d'un nouveau modèle pour les relations postcoloniales $^{7}$ à travers la mise en œuvre d'une politique de coopération qui se voulait exemplaire. En 1962, la fin de la période coloniale ne modifie pas immédiatement les caractéristiques de la présence française en Algérie. À l'Université, "l'œuvre de la France» d'avant 1962 n'avait concerné qu'une faible partie de la population de l'Algérie, pour l'essentiel des enfants d'européens nés ou installés sur place. Après le départ des Européens, le nombre d'enseignants et d'étudiants est dérisoire. La priorité est alors accordée à la formation car l'ouverture de l'Université aux étudiants algériens date seulement des années $1960^{\circ}$. Les centres de recherches héritées de l'ancienne puissance coloniale continuent de fonctionner dans le cadre d'un organisme provisoire de coopération scientifique dont la co-gestion est assurée par les États algérien et français, l'ocs. Durant cette période, la coopération franco-algérienne, plutôt déséquilibrée et tendue, porte essentiellement sur la formation de compétences scientifiques et techniques nécessaires à la reprise et au développement de l'Université et la poursuite de projets de recherche initiés avant l'indépendance. En réalité, malgré un discours-programme particulièrement ambitieux, l'activité scientifique est le fait de quelques initiatives personnelles, d'enseignants-chercheurs pour la plupart européens restés en Algérie après l'accession du pays à son indépendance, domiciliés au sein de quelques instituts (l'Institut d'études nucléaires, l'Institut océanographique, le Centre anti-cancéreux, le centre Pierre et Marie Curie et le Centre de recherche anthropologique et de préhistoire). Mais les urgences sont ailleurs. La recherche scientifique fait figure ici de portion congrue.

10 Au début des années 1970, les pouvoirs publics prennent conscience du rôle de la science et de la technologie dans le processus de développement. La recherche scientifique commence alors à être un véritable sujet de préoccupation pour les décideurs algériens. La recherche scientifique est délibérément intégrée dans les décisions gouvernementales dans ses divers domaines d'intervention. Au niveau institutionnel, la première décision ayant une portée hautement symbolique se traduit par la dissolution, en 1968, de l'OCS. Dans le même temps, on assiste à la formulation d'une nouvelle politique de la recherche scientifique.

Le nouveau dispositif institutionnel

11 En 1973, le remplacement de «l'Organisme algéro-français de coopération scientifique » (OCS) par un Conseil provisoire de la recherche scientifique algérien 
(CPRS) marquait symboliquement la «nationalisation " ${ }^{9}$ de la recherche scientifique et technique. La même année, le ministère de l'Enseignement supérieur et de la Recherche scientifique, une fois les bases de la réforme de l'enseignement supérieur jetées, s'attachait à l'organisation de son indispensable corollaire : la recherche scientifique.

Le débat inauguré en liaison avec les secteurs économiques aboutit à la création, en juillet 1973, de l'Organisme national de la recherche scientifique (ONRS) et du Conseil national de la recherche (CNR). En 1973, l'université algérienne comptait essentiellement trois universités, Alger, Oran et la toute jeune université de Constantine (créée en 1969). Celle d'Alger, de loin la plus importante, comptait 16000 étudiants. La recherche n'était donc le fait que de quelques centaines d'enseignants souvent en cours de thèses. Dans ce contexte, les missions de l'ONRS ont été principalement orientées dans deux directions : l'impulsion de la recherche dans les universités, et la liaison et la coordination de cette recherche avec le secteur socioéconomique.

13 Très vite, l'ONRS s'attache à développer la recherche universitaire en la distribuant selon deux grands volets: les instituts universitaires, parties intégrantes des universités et les centres de recherche, placés directement sous son contrôle.

L'ONRS a eu pour principal souci la formation du personnel chercheur. De 1974 jusqu'à sa dissolution en 1983, cet organisme a soutenu 109 projets de recherche dans les universités et 62 programmes.

L'évolution du potentiel chercheur est retracée dans le tableau suivant :

De même, 1340 publications furent réalisées, en quantité équivalente dans les revues nationales et internationales, témoignant du démarrage effectif de la recherche nationale.

lution de l'ONRS ${ }^{10}$ marqua un tournant dans le développement du processus de changements institutionnels de la recherche scientifique. Ses attributions et activités furent transférées. Il est inauguré le 7 juillet 1984, avec la création du Commissariat à la recherche scientifique et technique (CRST), rattaché au Premier ministre. Très vite, cette nouvelle structure met en place un Comité intersectoriel de coordination et de planification qui apparut comme l'organe privilégié d'une approche intégrée de la recherche, mettant à contribution tous les opérateurs concernés par un même domaine. Le CRST apparut historiquement comme la première instance à avoir pu dégager un certain nombre de programmes nationaux prioritaires en ajustant l'offre et la demande de recherche. Des groupes de concertation furent mis en place avec comme objectif d'assurer la convergence des programmes au niveau national. C'est ainsi que différents programmes furent élaborés dans les domaines suivants : mines, phosphates, métallurgie, fonderie, soudure, microélectronique, agronomie, technologie, agroalimentaire, zones arides et steppiques, hydrocarbures, industries chimiques, produits pharmaceutiques, pollution, sciences de l'éducation.

18 La restructuration de la recherche universitaire s'effectua en liaison avec le CRST et prit un essor nouveau à la faveur du travail entrepris par les groupes de concertation, des retours de formation de l'étranger et de la politique intense des laboratoires de recherche qui fut menée durant le Plan quinquennal 1980-1984 ${ }^{11}$. Malheureusement, l'absence de mise en place d'instruments financiers de régulation ne permit pas le parachèvement d'un processus institutionnel prometteur et durable. 
19 En 1986, de nouveaux changements institutionnels sont opérés et se traduisent par la dissolution du $\mathrm{CRST}^{12}$ et la création du Haut Commissariat à la recherche (HCR), placé auprès de la présidence de la République. Deux missions principales lui sont confiées : une mission verticale (développement des énergies nouvelles avec la possibilité de créer des centres de recherche dans d'autres domaines; une mission horizontale (mise en œuvre d'une politique d'animation et de coordination de la recherche scientifique nationale). Cependant, les attributions qui lui furent confiées (gestion du secteur de la recherche dite sensible d'une part et coordination d'autre part) furent trop ambitieuses et trop hétérogènes. Aussi, malgré quelques succès, le HCR ne pouvait mener de front l'ensemble du programme d'actions qui lui incombait. On a pu constater un fléchissement du rythme des coordinations intersectorielles. Le HCR souffrit en outre du même handicap que le CRST, en l'occurrence, l'absence d'une enveloppe recherche qui lui aurait probablement permis de mieux réguler et contrôler l'exécution des programmes de recherche à l'échelle nationale.

20 Cependant, compte tenu de sa vocation ou de son caractère exclusivement universitaire, l'ONRS n'a pas réussi à générer une dynamique de liaison avec les secteurs utilisateurs de la recherche pour différentes raisons développées ailleurs ${ }^{13}$.

Néanmoins, l'ONRS eut le mérite de lancer la recherche universitaire et contribua notablement à la formation à la recherche (plus de 200 thèses furent soutenues dans le cadre des projets financés) et à la mise sur pied d'un nombre important de laboratoires de recherche universitaires.

22 À l'Université, l'organisation et la gestion de la recherche universitaire, de type administratif et bureaucratique, ne s'améliorèrent guère, ce qui eut pour effet de freiner l'exécution des programmes de recherche. En l'absence d'une organisation pédagogique normalisée, la mise en place de départements pédagogiques et de recherche au sein des Instituts universitaires tarda à se mettre en place et ne sembla plus constituer une solution.

23 Ainsi, ce bref aperçu historique de l'évolution du système de la recherche scientifique en Algérie met en lumière un certain nombre de faits saillants. Il permet de mieux appréhender la situation actuelle et de préciser les termes d'une problématique plus globale de la recherche scientifique et technique et, du même coup, d'inscrire le processus d'émergence et de développement d'une communauté de chimistes qui constitue le vif de notre propos ici.

24 Un effort financier important est consenti par l'État-providence ${ }^{14}$ pour la mise en œuvre de la réforme de 1971, le développement du réseau universitaire et la formation de compétences scientifiques et techniques. Le montant des ressources allouées connaît une évolution stable.

De nouvelles universités voient le jour. Elles ont une vocation scientifique et technique clairement affichée. La plus importante d'entre elles, l'université de Bab-Ezzouar, construite grâce au concours de l'architecte brésilien Oscar Niemeyer, est située dans la banlieue est d'Alger. Très rapidement, elle deviendra le premier pôle scientifique et technologique du pays avec plus de 20000 étudiants et plusieurs centaines d'enseignants-chercheurs répartis dans toutes les disciplines des sciences dures: mathématiques, physique, chimie, biologie, sciences de la Terre, etc. De grands instituts sont également créés à travers le pays dans les sciences et les techniques. 

massification de l'accès au savoir universitaire. L'élargissement du recrutement social favorise l'arrivée d'étudiants issus de toutes les couches sociales, y compris des milieux les plus défavorisés. À titre d'exemple, le nombre des étudiants inscrits à l'université algérienne augmente de façon très nette, entre 1971 et 1980 passant de 23413 à 66064 . Les filles sont les principales bénéficiaires de cette démocratisation de l'accès aux études supérieures. Les effectifs étudiants féminins augmentent aussi considérablement passant de 5334 en 1971 à 18092 en 1980 et à 45000 en 1986.

Il mérite d'être souligné ici que pendant toute la période de gestation et de développement initial de l'université algérienne, le partenaire français a joué un rôle très précieux dans la formation des futurs maitres de conférences et professeurs d'une part et dans la mise en place de laboratoires de recherche. Ce point fera l'objet d'une étude ultérieure. Les laboratoires du CNRS ont joué un rôle particulièrement important. Par exemple, avec le CNRS, dont une représentation avait été instituée à Alger dès le début des années 1940, les liens scientifiques inaugurés durant la période coloniale ont perduré après l'accession de l'Algérie à son indépendance. Ils ont été confirmés par l'accord signé en 1975 avec l'ONRS et celui signé le 4 mars 1985 avec la Direction de la recherche scientifique du ministère de l'Enseignement supérieur et de la Recherche scientifique. Les sciences pour l'ingénieur, la physique et la chimie ont toujours constitué les disciplines forces de la coopération scientifique avec le CNRS. Et c'est en chimie (en catalyse notamment) que le premier programme international de coopération scientifique (Pics) fut institué dans les années 1990.

L'émergence d'une communauté de chimistes

1962, les Algériens prennent possession de l'université coloniale. La politique de la terre brûlée a laissé ses empreintes : incendie de la bibliothèque de l'université, départ massif des enseignants européens qui animaient cette université. Les enseignements redémarrent grâce à la coopération internationale et aux premiers coopérants qui répondent à l'appel de l'Algérie, au titre de leur engagement politique ${ }^{15}$. Dès la rentrée universitaire 1962-1963, à l'appel lancé par la nouvelle équipe dirigeante, le recrutement d'étudiants est massif. Mais il montre très vite une grande hétérogénéité des lieux comme des itinéraires de formation. Parmi eux, des expatriés, des militants dont les études avaient été interrompues par l'action politique, et ceux qui ont pu bénéficier de conditions spéciales, dérogatoires d'accès aux études supérieures.

Le redémarrage de la faculté des sciences d'Alger à l'indépendance

29 rappeler ici que, du XIX ${ }^{e}$ siècle à la Première Guerre mondiale, les Algériens étaient exclus des carrières scientifiques? Ainsi, l'histoire de la faculté des sciences d'Alger, tout au long de la période coloniale, révèle une institution « dont le rôle réel est de reproduire des rapports de domination et dont les enseignements dispensés expriment les intérêts d'une classe dominante ${ }^{16}$. Dans ce contexte, on comprend aisément que des disciplines telles que la médecine ou le droit aient pu occuper une place et un rôle de premier plan. Les choses n'évolueront qu'avec l'indépendance.

En pratique, la faculté des sciences d'Alger reste, jusqu'au début des années 1970, dominée par la présence française tant institutionnelle qu'universitaire. Dans les premières années de l'indépendance, son premier doyen est le professeur François Dumontet. Puis, de 1964 à 1970, Rachid Touri, le premier Algérien agrégé en mathématiques avant l'indépendance, lui succède.

La revue pour l'histoire du CNRS, 12 | 2005 
31 Au moment de l'indépendance, les enseignants de la faculté des sciences d'Alger, toutes disciplines confondues, sont, pour quelques-uns, des enseignants issus de familles " pieds-noirs » d'Algérie et qui y sont restées après 1962 ou des enseignants venus de l'ex-métropole. Parmi ces enseignants, des communistes qui avaient marqué leur engagement en faveur de l'indépendance de l'Algérie tel le professeur Robert Sauterey, normalien de la promotion 1943, et des chrétiens libéraux. Ici, le cas d'André Mandouze a valeur d'exemple ${ }^{17}$. Universitaire, latiniste de renom, il est le premier directeur de l'enseignement supérieur en Algérie ${ }^{18}$.

La création du département de chimie

Quelques changements institutionnels intervinrent dès les premières années de l'indépendance.

33 En 1966, un chimiste algérien, Ramdane Ouahes, arrive à Alger. Agrégé de l'École normale de la rue d'Ulm, il est, dès son retour, porté par ses collègues algériens pour succéder à Robert Sauterey à la tête de la chaire de chimie, avec l'idée d'une réorganisation institutionnelle de la chimie à l'Université. Un projet de création d'un véritable département de chimie à l'université d'Alger se profile. Étant le plus gradé parmi ses collègues algériens, Ramdane Ouahes prend la direction du département de chimie créé en 1964.

Après l'indépendance, plusieurs coopérants français arrivent à Alger pour renforcer la communauté naissante. On peut citer, par ordre alphabétique, des professeurs: Micheline Broust-Bournazel, Annie Diara, Valentin Hekanel; des maitres de conférences : Marc J. M. Abadie, Daniel Bodiot, Jean-Bernard Bourdet, Michel Daguenet, José Gayozo, Jean-Pierre Monthexerd, Louis Robert, Louis Schuffenecker ${ }^{19}$, Bernard Spinnck, Jean-Maurice Vergnaud, Edgard Wendling ${ }^{20}$; des maîtres-assistants : Bernard Devallez, Michel Andriaux, André Pralloux, Robert Granger, Michel Leard, ainsi que des volontaires du service national actif (VSNA) tels Serge Monteverdi ou Jean-Yves Sanchez ${ }^{21}$.

L'institutionnalisation d'un département de chimie et le renforcement de la communauté universitaire, parallèlement à la mise en oeuvre de l'accord francoalgérien de 1963, vont susciter, en 1965, la venue d'une deuxième vague d'étudiants algériens composée de physiciens, de chimistes, de mathématiciens, tous titulaires de thèses de troisième cycle. Ces étudiants, parmi lesquels on comptait des militants de l'Union générale des étudiants musulmans algériens (Ugema) ${ }^{22}$ étaient en majorité issus de l'université de Bordeaux alors que ceux de la première vague, qui a caractérisé l'année 1963, étaient partagés entre les universités de Besançon et Dijon. Parmi eux, on peut citer les noms de Abdelkader Kacher, Mustapha Bouhadef et Hakim Ladjouze. Les jeunes assistants qui ont assuré le démarrage de l'université à l'indépendance, en l'occurrence Mouloud Achour, M'hamed Meklati, Ouassini Benali-Baïtich, préparent, de leur côté, leur thèse.

De façon générale, la plupart des thèmes de recherche abordés relèvent davantage de la science fondamentale ${ }^{23}$ et servent plus particulièrement à l'organisation de formations supérieures spécialisées sans prise sur le secteur économique. Il faudra attendre la réforme qui touchera l'Université algérienne à partir de 1970 pour voir se profiler de nouvelles logiques académiques et la volonté manifeste des pouvoirs publics d'instaurer de nouveaux liens entre science et industrie.

La Société algérienne de chimie, une première dans le paysage scientifique de l'Algérie indépendanteLes origines 
37 L'idée de créer une société savante dans le domaine de la chimie avait germé depuis de nombreuses années dans les milieux scientifiques algériens. Mais c'est seulement en novembre 1988 que l'idée va se concrétiser. En effet, à cette date, se tient à Oran ( $2^{\mathrm{e}}$ ville d'Algérie, située à environ $500 \mathrm{~km}$ de la capitale) le troisième séminaire national de chimie. Et lors de cette rencontre scientifique, une réunion est organisée à l'initiative de son comité d'organisation. C'est dans ce cadre qu'un groupe de chimistes se concerte et envisage la création d'une Société algérienne de chimie afin de combler le vide scientifique dans ce domaine. Ce groupe est initialement composé d'une douzaine d'universitaires, représentant les différentes spécialités de la chimie (chimie organique, minérale, physique, théorique). Parmi eux, il faut citer les professeurs Abdou Boucekkine, Mohammed El Miloud Bettahar, Hacène Kerdjoudj (rattachés à l'université des sciences et des techniques d'Alger) ${ }^{24}$, Hadj Benhaoua et Abdelkader Bensadat (de l'université d'Oran Es Sénia) et de deux femmes, Djamila Harrache (maître-assistante à Sidi-Bel-Abbès) et Reghia Bencheriet (maître-assistante à l'université de Constantine) $)^{25}$.

38 Le projet intervient dans un contexte social, économique et politique marqué par la très grave crise de 1988 et les émeutes qui avaient éclaté en Algérie un mois auparavant. Avant de décrire cette société, il convient de rappeler brièvement les conditions sociopolitiques qui entourent l'émergence de cette société savante.

La discussion porta d'abord sur les objectifs à assigner à cette association scientifique : il s'agissait de créer une organisation scientifique qui devait être autonome, en opposition avec toutes les tentatives antérieures instituées sous l'égide du FLN. Pour cela, il fallait faire agréer ses statuts, son programme, créer un bulletin d'information et un journal scientifique centrés sur la vie de l'association, établir des relations avec les secteurs économiques nationaux, les partenaires scientifiques étrangers et promouvoir la recherche dans les différentes spécialités de la chimie. Dans ce contexte malgré tout dominé institutionnellement par le parti FLN alors au pouvoir (malgré son affaiblissement, sa perte de légitimité et sa remise en cause récente), l'entreprise n'est pas simple. Mais le projet se concrétise finalement dans un climat un peu plus propice. En effet, un vent de liberté et de démocratie commence à souffler sur le pays, ce qui encourage cette entreprise. Le projet s'inscrit à la fois dans les mesures récentes relatives aux associations ${ }^{26}$ et dans le mouvement de démocratisation politique qui traverse l'Algérie au lendemain des émeutes d'octobre 1988. Une nouvelle constitution est votée en février 1989 ; en juillet de la même année, l'Assemblée populaire nationale vote la loi qui légalise le pluripartisme ; dans la foulée, le Front islamique du salut (FIS) est légalisé et légitimé ; la presse est libéralisée et les associations se développent avec, parmi elles, de nombreuses associations féminines.

40 C'est dans ce climat d'apparente démocratisation, marqué de fait par l'essor de l'islamisme radical, que la première société savante tant attendue des chimistes va naître.

La création de la Société

41 Le groupe précité s'associe pour fonder la Société algérienne de chimie (dorénavant Sac dans le texte) lors de l'Assemblée générale constitutive qui se tient à Alger le 27 mars 1989 à l'université des sciences et des techniques, au siège de l'Institut de chimie. 98 chimistes appartenant aux différentes institutions algériennes de formation supérieure ou de recherche scientifique, forment l'Assemblée générale constitutive de la Sac, dont la présidence est confiée au professeur Ramdane Ouahes de l'USTHB, le plus âgé d'entre 
eux. Les participants sont déclarés membres fondateurs. Au terme de cette assemblée générale constitutive, un bureau composé de 11 membres, chargé de la mise en place de l'association et du démarrage de ses activités, est élu. Il est composé d'un président: Abdou Boucekkine, professeur de chimie à l'USTHB et de trois vice-présidents : Hadj Benhaoua, professeur de chimie à l'université d'Oran es-Sénia, élu vice-président de la région Ouest; Mohammed El Miloud Bettahar, professeur de chimie à l'USTHB, élu viceprésident de la région Centre; Abdelkrim Haouam, maître de conférences en chimie à l'université de Constantine, élu vice-président de la région Est. Un programme est voté. La Sac est créée par l'arrêté ministériel du 18 juin $1989^{27}$. Le siège social est installé à Alger. Dès sa création, les statuts de la Sac stipulent l'organisation d'un congrès tous les deux ans.

$\mathrm{Au}$ moment de sa fondation, la Sac compte une centaine de membres. Certaines des personnalités scientifiques qui y sont représentées, appartiennent parfois à des sensibilités politiques divergentes. La Sac est un microcosme et les clivages sousjacents, révélateurs de ceux qui traversent le corps social, y sont de plusieurs ordres :

- politique : les chimistes qui adhèrent à la Sac ne forment pas un bloc monolithique. Ils représentent les différentes sensibilités qui traversent la société : le FLN (Front de libération nationale, ex-parti unique) et toutes les autres sensibilités tels le FFS (Front des forces socialistes), le PAGS (Parti de l'avant-garde socialiste, d'obédience communiste), et la mouvance islamiste (dans sa « diversité »);

- linguistique : schématiquement, les chercheurs qui adhèrent à la Sac sont de deux types : les francophones, majoritaires, et les arabophones, plus jeunes, formés dans le cadre d'une arabisation expérimentale lancée, dès les premières années de l'indépendance, en direction des sciences « dures ». Au-delà des conflits apparemment linguistiques, les défenseurs de l'une ou l'autre langue, en s'opposant sur ce terrain, reflètent en réalité les conflits idéologiques et politiques qui divisent « culturalistes » et « technocrates " ${ }^{28}$ et dont la finalité est une lutte pour la prise du pouvoir politique ;

- régionaliste : les clivages de cet ordre ont toujours existé en Algérie. L'existence de pôles identitaires régionaux n'est pas une construction paradigmatique récente.

Elle est largement antérieure à la colonisation française. Au XIV siècle, Ibn Khaldun, dans sa Muqaddima, ses Prolégomènes au Kitab al 'Ibar avait décrit le système social des populations du Maghreb central, leur hiérarchisation en groupes familiaux, claniques et tribaux et avait forgé les concepts qui fondent cette identité, la 'açabiyya, solidarité socio-agnatique caractéristique des groupes régionaux (régionalistes) contre toute menace d'intégration holiste qui leur ferait perdre leur appartenance communautaire. Et depuis la fin des années 1960, les processus de construction des identités professionnelles se heurtent à ces appartenances régionales, familiales, claniques, voire tribales. À ces oppositions ancestrales, viennent se greffer notamment des conflits entre la conception berbériste - représentée par les ardents défenseurs d'une affirmation identitaire berbère ${ }^{29}$ - et la conception arabo-islamique de l'Algérie - au sein de laquelle on retrouve les défenseurs de la langue arabe et de l'appartenance à la grande Umma islamique.

Dans le même temps, ces clivages qui apparaissent au grand jour, sont le signe de la fin d'une époque : celle du discours unanimiste qui prévalait jusqu'aux années 1980. La fondation de la Sac ne pouvait donc échapper à ces clivages. Mais elle est un creuset: malgré les clivages mentionnés ci-dessus, les scientifiques ont semble-t-il réussi à s'entendre autour du projet de fondation de cette société savante. 
45 La plupart du corps professoral qui compose la Sac a été formé à l'étranger ou en relation avec l'étranger. Certains ont soutenu leur thèse à Alger mais l'ont préparé grâce au soutien de la coopération scientifique internationale et tout particulièrement française. Parmi eux, Abdou Boucekkine a soutenu sa thèse à Alger mais il a été formé dans le cadre de la coopération scientifique franco-algérienne. D'autres ont eu un parcours universitaire exclusivement français. Parmi ces derniers, il est possible de mentionner que le doyen des chimistes, Ramdane Ouahes est normalien issu de l'ENS de Saint-Cloud; Mohammed El Miloud Bettahar a suivi toutes ses études supérieures en France où il a soutenu une thèse de doctorat à l'université d'Orsay et démarré sa carrière scientifique au sein du CNRS à Paris en 1969 avant de regagner l'Algérie en 1974 au moment de la création de l'université des sciences et des techniques de BabEzzouar.

Les sources de financement de la Sac

46 La Sac est financée par les cotisations de ses adhérents et les subventions accordées par le ministère délégué aux Universités et le ministère de l'Information et de la Communication, mais surtout grâce aux sponsors industriels et des institutions ainsi qu'aux parrainages occasionnels lors de l'organisation de manifestations scientifiques ${ }^{30}$. Ces financements couvrent les frais occasionnés par la publication du journal et du bulletin d'information mais aussi l'organisation des rencontres scientifiques.

47 La subvention accordée par le ministère de l'Enseignement supérieur et de la Recherche est aléatoire, faite de « zones d'incertitudes ». De manière générale, face aux restrictions budgétaires imposées par les organisations étatiques, les scientifiques doivent «se battre » régulièrement pour l'obtention de cette subvention, car elle ne leur est jamais acquise. De fait, la Sac vit surtout des recettes issues des cotisations de ses membres.

Le Journal de la Société algérienne de chimie

48 Deux ans après sa création, la Sac publie le premier numéro de son journal scientifique, le Journal de la Société algérienne de chimie.

49 Trente ans après l'indépendance, ce journal est apparemment le premier du genre en Algérie et reflète bien l'absence de journaux scientifiques avant l'indépendance (hormis le Bulletin de la Société d'histoire naturelle d'Afrique du Nord) ${ }^{31}$. Avant 1989, les performances des chimistes étaient publiées essentiellement à l'étranger, et plus particulièrement en France. Avec la création de ce journal, les chimistes marquent leur désir de favoriser les conditions d'émergence d'une production scientifique, loin des cénacles de la "grande science", en privilégiant sa visibilité localement, sans pour autant en sacrifier la qualité. Le journal paraît deux fois par an et cette périodicité est respectée, même entre 1991 et 1996, malgré de nombreuses difficultés. Le comité de rédaction est composé de professeurs appartenant tous à la plus grande université des sciences et des techniques de la banlieue algéroise. L'orientation du journal porte sur la recherche fondamentale ou appliquée. Les articles présentent des résultats expérimentaux originaux se rapportant à des travaux de recherche dans le domaine de la chimie (des mémoires de 10 pages, des mises au point sur un sujet ou thème donné de 20 pages et des notes de laboratoire de 2 à 5 pages). Les articles sont acceptés dans les trois langues suivantes : arabe, français, anglais. La qualité du journal tient au fait qu'il est indexé aux Chemical Abstracts, la référence incontournable des publications scientifiques en chimie et au fait que les propositions d'articles sont soumises à un comité de lecture. Ce comité est élargi à des experts scientifiques internationaux (basés 
principalement en France). La participation d'experts étrangers à ce comité permet de pallier aux insuffisances de la masse critique algérienne en élargissant les compétences scientifiques du journal et de limiter les possibles querelles de personnes. La présence de ces experts donne en outre à la société et à son journal une dimension et une audience internationales ${ }^{32}$.

La production scientifique

Avant de décrire la production scientifique des chercheurs de la Sac et d'entrevoir les réseaux au sein desquels sont insérés les chimistes algériens, il est utile de rappeler que la dynamique scientifique engendrée par la création de la Sac intervient dans un climat social et politique peu propice à l'activité scientifique: grèves de juin 1991, état de siège, campagne législative, démission du président de la République, état d'urgence, assassinat du président du Haut Comité d'État, terrorisme et couvre-feu à Alger... Ce contexte, s'il a constitué un obstacle majeur au développement des activités de la Société (restrictions de l'organisation de réunions des Bureau et Conseil nationaux, des manifestations scientifiques, défection de membres du bureau, etc.), n'a pas pour autant empêché la production de travaux scientifiques.

51 Si l'on examine la production scientifique publiée dans le Journal de la Sac, on peut observer la coexistence d'au moins deux types de travaux: les uns sont proches $\mathrm{du}$ mainstream (big science), liés à la recherche fondamentale - certains d'entre eux sont indexés à la base américaine du SCI ; c'est le cas par exemple d'articles sur les modèles de diffusion solide-solide, sur les mécanismes réactionnels, sur les intermédiaires réactionnels, sur les études spectroscopiques; les autres sont plus localistes, plus orientés sur la recherche expérimentale ou soucieux de répondre à un besoin formulé par une entreprise économique. C'est le cas d'articles consacrés à la dégradation de la cellulose, à l'oxydation du métal ou à la corrosion.

Ainsi, la production scientifique témoigne de stratégies différentielles opérées par les chercheurs, soucieux tout à la fois de constituer une production scientifique locale et de s'orienter vers des revues internationales qui publient en français ou en anglais.

Entre 1991 et 1996, 113 articles ont été publiés par le Journal de la Sac.

Sur les 113 articles publiés, on a pu dénombrer 31 articles de recherche appliquée, soit $27,4 \%$ du total des articles.

Dans le cadre de la coopération internationale, pour la même période, 37 articles (soit $32,7 \%$ ) ont été co-signés par des chercheurs algériens et leurs partenaires étrangers, principalement français.

La Sac, des réseaux nationaux ou internationaux entre science et industrie

Dès sa création, la Sac s'était fixé pour objectif de développer des relations scientifiques avec l'industrie et les autres sociétés savantes.

Ouverte dès sa création aux industriels, le nombre de ceux-ci, estimé à $10 \%$, s'est accru progressivement au fil des années. C'est ainsi qu'on y trouve des représentants du secteur des hydrocarbures et de la pétrochimie (Sonatrach), de la pharmacie, des engrais (Asmidal) ${ }^{33}$, des industries du verre (Enava), des matériaux de construction (SNMC). Ce sont d'ailleurs ces secteurs qui financent en partie les congrès et toutes les autres manifestations scientifiques organisées par la Sac. Les industriels ont trouvé quelque intérêt à coopérer avec l'Université pour permettre à leurs cadres d'être en contact avec des chercheurs par l'intermédiaire de la Sac. Réciproquement, les chercheurs pouvaient avoir théoriquement accès à des informations industrielles et 
initier des projets à caractère appliqué. Dans la pratique, la recherche industrielle, qui constitue une part plus modeste de l'ensemble des activités de recherche scientifique, s'est contentée d'actions ponctuelles limitées au secteur de la chimie et du gaz naturel.

À l'échelle du monde arabe, la Sac adhère à la Fédération des associations des chimistes arabes. Mais en raison de difficultés à la fois financières, politiques et linguistiques, les échanges scientifiques sont restés symboliques.

Au niveau maghrébin, la Sac a participé à la création des associations des chimistes maghrébins (Fès, 1992). Mais malgré la relance du Grand Maghreb dans le cadre de l'Union du Maghreb arabe (Uma) et le contexte des relations intermaghrébines, cet ensemble n'a pas non plus donné tous les résultats escomptés. La Sac entretient cependant des relations plus régulières avec la société tunisienne.

$\mathrm{Au}$ niveau européen, des relations scientifiques ont lieu avec des pays tels l'Allemagne, la Géorgie, la Pologne, la Roumanie. De fait, des relations plus suivies sont entretenues avec la Société française de chimie $(\mathrm{SFC})^{34}$ et de manière plus spécifique avec certaines sections de cette société (en catalyse, polymères).

Le CNRS a contribué au financement des quatre colloques franco-maghrébins de catalyse organisés par la $\mathrm{Sac}^{35}$.

Conclusions

62 En Algérie, la création récente d'une société savante de chimistes est une première. Son émergence s'inscrit au début des années 1990 dans un contexte marqué par une grave crise intérieure. L'activité scientifique, inséparable de son environnement, en subit les contrecoups. Dans cette perspective, l'exemple des chimistes algériens est particulièrement intéressant. Malgré un contexte d'étatisation et de bureaucratisation de la science, les chimistes ont pu se doter d'une société savante et d'une revue spécialisée. Leur dynamisme et leur professionnalisme représente une étape majeure dans l'émergence et le développement d'une communauté scientifique et la construction d'une identité sociale reconnue qui définit l'existence sociale du groupe et lui permet d'être pensé par les autres institutions qui composent le champ social. La création d'une société savante dans un contexte politique peu propice à l'activité scientifique est l'oeuvre d'enseignants-chercheurs appartenant à l'un des corps les plus professionnalisés. Cette société et son journal consacrent la ténacité de scientifiques convaincus de la première heure. Pour nombre d'entre eux, cette institution scientifique, née dans un contexte de violence politique extrême, est un défi lancé par quelques individualités afin de renforcer les liens au sein de la communauté scientifique locale et d'entretenir les réseaux de sociabilité scientifique malgré les vicissitudes de l'environnement scientifique local.

\section{NOTES}

1.Jean-Marie Lehn, Construire l'avenir - Livre blanc sur la Recherche présenté au Président de la République, Paris, La Documentation française, 1980, p. 263-291, cité in Micheline Charpentier-Morize, «La Contribution des "Laboratoires Propres" du CNRS à la 
Recherche chimique en France de 1939 à 1973 ", Cahiers pour l'Histoire du CNRS, Paris, CNRS ÉDITIONS, 1989-4, p. 82.

2.L'université d'Alger, créée en 1909, fut la première institution académique fondée par l'empire colonial français dans ses colonies.

3.En outre, il convient de souligner que le système colonial n'avait implanté d'industries que pour répondre au marché de la minorité coloniale, essentiellement pour transformer les produits agricoles.

À cela il faut ajouter que la concurrence des industries européennes porta un coup aux petites industries locales. C'est seulement après la Seconde Guerre mondiale avec la découverte et l'exploitation des premiers gisements d'hydrocarbures que les projets de développement commencèrent à éclore mais le mouvement pour l'indépendance avait entrepris le processus de décolonisation.

4.Avec les pays arabes, les relations devinrent plus soutenues au moment du lancement de la politique d'arabisation (années 1980) tandis que la coopération avec les ex-pays socialistes porta essentiellement sur les aspects militaires et politiques (avec des tentatives dans la formation des ingénieurs) et celle entretenue avec les pays anglosaxons se focalisa sur l'exploitation du pétrole et du gaz naturel.

5.Cf. la réforme de l'enseignement supérieur, Alger, MESRS, 1971 et l'ouvrage de A. Henni, La Mise en cuvre de l'option scientifique et technique, Alger, Cread, 1987.

6.Jean-Jacques Salomon, Science et politique, Paris, Economica, 1989, p. 158 et suiv. 7.Cf. notamment les travaux de William Zartman, « Les Relations entre la France et l'Algérie », Revue française de science politique, 6 décembre 1964, p. 1107-1108 ; Nicole Grimaud, La Politique extérieure de l'Algérie, Paris, Karthala, 1984 ; Salah Mouhoubi, La Politique de coopération algéro-française : bilan et perspectives, Paris/Alger, Publisud/Opu, 1986 ; Jean-François Daguzan, « Les Rapports franco-algériens, 1962-1992 », Politique étrangère, hiver 1993-1994, p. 885-896.

8.En 1909, l'université d'Alger est créée pour former les enfants d'européens installés en Algérie. Les Algériens musulmans sont faiblement concernés par cet enseignement supérieur. Pour plus de détails, cf. G. Pervillé, Les Étudiants algériens de l'université française. 1880-1962, Paris, CNRS Éditions, 1984.

9.Cf. La « nationalisation du pétrole».

10.Cette dissolution fut consacrée par le décret du 17 décembre 1983.

11. Cf. notamment MERS/ONRS, Deuxième rapport Bilan sur les activités de recherche de l'ONRS, Alger, 1982.

12.Cf. décret du 28 août 1986.

13.Y. Bettahar, « La Société d'histoire naturelle d'Afrique du Nord. Algérianisation d'une société savante coloniale ", Sciences, savoirs modernes et pouvoirs dans le monde musulman contemporain, Revue des mondes musulmans et de la Méditerranée, $\mathrm{n}^{\circ}$ 101-102, 2003, p. 157-173.

14.De façon générale, la rente pétrolière, grâce à la « nationalisation » en 1971 des hydrocarbures, favorise le mouvement de réformes qui est entrepris durant cette période.

15.Parmi eux, des Français, appelés communément " pieds rouges ».

16.Comme a déjà pu l'observer le sociologue Jean-Michel Berthelot pour l'École en France.

17.Cf. André Mandouze, Mémoires d'outre-siècle. D’une résistance à l'autre, Tome I, Paris, Éditions Viviane Hamy, 1998 ; Mémoires d'outre-siècle, Tome II, 1962-1981, Paris, Éditions du Cerf, 2003. 
18.Puis lui succéderont notamment Youcef Mentalechta (1966-1968), Hamid Bensalem (1968-1970) et Rachid Touri (1970-1980).

19.L. Schuffenecker séjourna en Algérie de 1976 à 1984. Il fut le principal initiateur et animateur de l'enseignement de la thermodynamique à l'université des sciences et des techniques de Bab-Ezzouar (banlieue est d'Alger). Très tôt, il s'entoure d'une équipe de recherche et contribue ainsi à la formation de docteurs algériens et français (VSNA). Il est actuellement président de l'Institut national polytechnique de Lorraine (INPL).

20.Nous remercions vivement les universitaires chimistes algériens et français qui ont accepté de répondre à nos questions lors des entretiens effectués à Alger et à Nancy. 21.Une étude plus détaillée relative aux coopérants français ayant séjourné en Algérie entre 1960 et 1980 est en cours.

22.Cette organisation, fondée en 1955 à la veille de l'appel du FLN, s'est chargée de l'envoi en formation à l'étranger, d'étudiants algériens ayant quitté les bancs de l'Université française en 1956.

23.Par exemple sur le champ cristallin, un sujet de pointe pour l'époque, traité en collaboration avec un professeur danois.

24.Dorénavant USTHB dans le texte.

25. Cf. Société Algérienne de Chimie, Bulletin intérieur, n 1, novembre 1989, p. 3-4.

26.Se reporter au décret $n^{\circ} 88 / 16$ du 2 février 1988 fixant les modalités d'application de la loi $\mathrm{n}^{\circ} 87 / 15 \mathrm{du} 21$ juillet 1987 relative aux associations, ainsi qu'aux dispositions statutaires communes aux associations.

27. Cet arrêté, portant agrément de l'Association nationale dénommée " Société algérienne de chimie », est publié au Journal officiel du 12 juillet 1989.

28. Nous utilisons ces appellations par simple commodité. Mais il convient de signaler qu'elles ne nous satisfont guère car au-delà de cette réduction simplificatrice, les conflits autour de la langue nous paraissent autrement plus complexes.

29.Qui a trouvé son mode d'expression à travers notamment la crise dite « berbériste » de 1949, le « printemps berbère » des années 1980 et le « mouvement culturel

berbère ». Ce mouvement, fortement contestataire, est bien représenté dans les milieux universitaires.

30.Parmi ces institutions figurent à l'occasion le secrétariat d'État à la Recherche, l'Office national de tourisme, les universités régionales et les centres de recherche dépendant des ministères techniques (Cerhyd, CRD) et d'entreprises nationales (Sonatrach, Diprochim, Enad, Asmidal, etc.), le service scientifique près l'Ambassade de France en Algérie (à l'occasion du $2^{\mathrm{e}}$ Congrès franco-maghrébin).

31.Cf. à ce sujet, Y. Bettahar, «L'Algérianisation d'une société savante coloniale », 2003, op. cit.

32.Il convient de signaler que la British Library of London et la bibliothèque de Hanovre en Allemagne figurent parmi les abonnés.

33. Asmidal effectue des recherches appliquées dont la finalité est la maîtrise des procédés importés et la compréhension des problèmes de génie chimique pour la production d'ammoniac.

34. Comparée à notre jeune société, la Société française de chimie a un long passé derrière elle comme le montrent les travaux de B. Bensaude-Vincent et I. Stengers, Histoire de la chimie, Paris, La Découverte, 1995.

35.Colloques franco-maghrébins organisés à Hammamet (Tunisie) en 1990, à Sidi-Fredj (Algérie) en 1992, à Rabat (Maroc) en 1994 et à Lille (France) en 1996. 
INDEX

Mots-clés : Société algérienne de chimie, chimie, Algérie

\section{AUTEUR}

YAMINA BETTAHAR

Yamina Bettahar est maître de conférences à l'INPL/ENSGSI et chercheur au LPHS Archives Henri Poincaré, UMR nº 7117 du CNRS, université Nancy 2. 